\title{
Nodal Discontinuous Galerkin Method for Time-Domain Lorentz Model Equations in Meta-Materials
}

\author{
Shanghui $\mathrm{Jia}^{1}$, Changhui Yao ${ }^{2, *}$ and Shuai $\mathrm{Su}^{3}$ \\ ${ }^{1}$ School of Statistic and Mathematics, Central University of Finance and \\ Economics, Beijing 100081, China \\ ${ }^{2}$ School of Mathematics and Statistics, Zhengzhou University, Zhengzhou, China \\ ${ }^{3}$ Graduate School of China Academy of Engeering Physics, Beijing 100088, China
}

Received 8 January 2016; Accepted (in revised version) 19 July 2016

\begin{abstract}
In this paper, Nodal discontinuous Galerkin method is presented to approximate Time-domain Lorentz model equations in meta-materials. The upwind flux is chosen in spatial discrete scheme. Low-storage five-stage fourth-order explicit Runge-Kutta method is employed in time discrete scheme. An error estimate of accuracy $\mathscr{O}\left(\tau^{4}+h^{n}\right)$ is proved under the $L^{2}$-norm, specially $\mathscr{O}\left(\tau^{4}+h^{n+1}\right)$ can be obtained. Numerical experiments for transverse electric (TE) case and transverse magnetic (TM) case are demonstrated to verify the stability and the efficiency of the method in low and higher wave frequency.
\end{abstract}

AMS subject classifications: 35L05, 76M10

Key words: Time-domain Lorentz model, meta-materials, Runge-Kutta method, nodal discontinuous Galerkin method.

\section{Introduction}

The discontinuous Galerkin (DG) method [1] has gained more popularity in solving various differential equations [2-6] in recent years for its great flexibility in mesh construction and its convenience in parallel implementation. During the past years, Many DG researches were explored for electromagnetic systems in the free space [7-14] and dispersive media [15-17] whose permittivity depended on the wave frequency. Very recently, the DG method was developed to solve Maxwell's equations in meta-materials $[18,19]$.

The meta-materials $[20,21]$ are artificially structured electromagnetic nano-materials with some exotic properties such as negative refraction index and amplification of evanescent waves. The advantages of meta-materials mainly come from their potential applications in diverse areas such as building a perfect lens, sub-wavelength imaging and cloaking. In the past decade, engineers and physicists had been engaged in many numerical simulations for Maxwell's equations in meta-materials. However, such simulations were almost ${ }^{*}$ Corresponding author. Email addresses: shjia@lsec.cc.ac.cn (S. H. Jia), chyao@lsec.cc.ac.cn
(C. H. Yao), sushuaiby@sina.cn (S. A. Su) 
established on either the classic finite-difference time-domain (FDTD) method or some commercial software packages with constraints and limitations. In recent years, some effort $[18,20-22,24]$ in developing and analyzing some finite element methods(FEMs) for time-domain Maxwell's equations involving meta-materials were established.

In [21], the author proved stability, existence and uniqueness of the solution for the Lorentz model equations. In [31], the author provided the existence and uniqueness for a vector wave integro-differential equation. In [32], the author provided stability for the single pole Debye medium model equations.

In [17], A discontinuous Galerkin method for the numerical approximation of timedependent Maxwell equations in three different dispersive media was introduced. Both the $L^{2}$-stability and error estimate of the DG method were discussed in detail. In [18], the author proposed a leap-frog discontinuous Galerkin method to solve the time-dependent Maxwell's equations in meta-materials. Conditional stability and error estimates were proved for the scheme. In [30], the author developed a nodal discontinuous Galerkin method for solving the time-dependent Maxwell's equations when meta-materials were involved. Both semi- and fully-discrete schemes were constructed. Numerical stability and error estimate were proved for both schemes.

In this paper, Nodal discontinuous Galerkin method is presented to approximate timedomain Lorentz model equations in meta-materials. The upwind flux is chosen in spatial discrete scheme. Low-storage five-stage fourth-order explicit Runge-Kutta method is employed in time discrete scheme. The energy is decreasing in time. An error estimate of accuracy $\mathscr{O}\left(\tau^{4}+h^{n}\right)$ is proved under the $L^{2}$-norm, specially $\mathscr{O}\left(\tau^{4}+h^{n+1}\right)$ can be obtained. Numerical experiments for TE and TM cases are demonstrated to verify the stability and the efficiency of the method in low and higher wave frequency. The content of this paper is summarized as follows. In Section 2, we present the governing equations for metamaterials and for deformation. In Section 3, We develop a DG method and conduct theory analysis. In Section 4, We get the semi-discrete DG method and use the classic low-storage five-stage fourth-order explicit Runge-Kutta method [28] for time discretization. Then in Section 5, we implement numerical results for the meta-material model and analyze large wavenumber.

\section{Time-domain Lorentz model}

Time-domain Lorentz model is described by the following governing equations

$$
\begin{array}{ll}
\varepsilon_{0} \frac{\partial \widetilde{\mathbf{E}}}{\partial \tilde{t}}+\frac{\partial \widetilde{\mathbf{P}}}{\partial \tilde{t}}-\nabla \times \widetilde{\mathbf{H}}=0, & (0, T] \times \Omega, \\
\mu_{0} \frac{\partial \widetilde{\mathbf{H}}}{\partial \tilde{t}}+\frac{\partial \widetilde{\mathbf{M}}}{\partial \tilde{t}}+\nabla \times \widetilde{\mathbf{E}}=0, & (0, T] \times \Omega, \\
\frac{1}{\varepsilon_{0} \tilde{\omega}_{p e}^{2}} \frac{\partial^{2} \widetilde{\mathbf{P}}}{\partial \tilde{t}^{2}}+\frac{\tilde{\Gamma}_{e}}{\varepsilon_{0} \tilde{\omega}_{p e}^{2}} \frac{\partial \widetilde{\mathbf{P}}}{\partial \tilde{t}}+\frac{\tilde{\omega}_{e 0}^{2}}{\varepsilon_{0} \tilde{\omega}_{p e}^{2}} \widetilde{\mathbf{P}}-\widetilde{\mathbf{E}}=0, & (0, T] \times \Omega,
\end{array}
$$




$$
\frac{1}{\mu_{0} \tilde{\omega}_{p m}^{2}} \frac{\partial^{2} \widetilde{\mathbf{M}}}{\partial \tilde{t}^{2}}+\frac{\tilde{\Gamma}_{m}}{\mu_{0} \tilde{\omega}_{p m}^{2}} \frac{\partial \widetilde{\mathbf{M}}}{\partial \tilde{t}}+\frac{\tilde{\omega}_{m 0}^{2}}{\mu_{0} \tilde{\omega}_{p m}^{2}} \widetilde{\mathbf{M}}-\widetilde{\mathbf{H}}=0, \quad(0, T] \times \Omega,
$$

where $\widetilde{\mathbf{E}}$ and $\widetilde{\mathbf{H}}$ are the electric and magnetic fields, respectively. $\widetilde{\mathbf{P}}$ and $\widetilde{\mathbf{M}}$ are the induced polarization and magnetization, respectively. $\omega_{0}$ and $\mu_{0}$ are the vacuum permittivity and permeability, respectively. $\tilde{\omega}_{p e}$ and $\tilde{\omega}_{p m}$ are the electric and magnetic plasma frequencies, respectively. $\tilde{\omega}_{e 0}$ and $\tilde{\omega}_{m 0}$ are the electric and magnetic resonance frequencies, respectively. $\tilde{\Gamma}_{e}$ and $\tilde{\Gamma}_{m}$ are the electric and magnetic damping frequencies, respectively. The induced electric and magnetic currents are described by $\widetilde{\mathbf{J}}=\frac{\partial \widetilde{\mathbf{P}}}{\partial \tilde{t}}$ and $\widetilde{\mathbf{K}}=\frac{\partial \widetilde{\mathbf{M}}}{\partial \tilde{t}}$, respectively.

To make the problem well posed, we simply assume that the boundary of $\Omega$ is perfect conducting:

$$
\hat{\mathbf{n}} \times \widetilde{\mathbf{E}}=0, \quad \text { on } \quad \partial \Omega \text {, }
$$

where $\hat{\mathbf{n}}$ is the unit outward normal to $\partial \Omega$. Furthermore, we assume that the initial conditions are

$$
\begin{array}{rlrl}
\widetilde{\mathbf{E}}(\tilde{x}, 0) & =\widetilde{\mathbf{E}}_{0}(\tilde{x}), & \widetilde{\mathbf{J}}(\tilde{x}, 0)=\widetilde{\mathbf{J}}_{0}(\tilde{x}), & \widetilde{\mathbf{P}}(\tilde{x}, 0)=\widetilde{\mathbf{P}}_{0}(\tilde{x}), \\
\widetilde{\mathbf{H}}(\tilde{x}, 0)=\widetilde{\mathbf{H}}_{0}(\tilde{x}), & \widetilde{\mathbf{K}}(\tilde{x}, 0)=\widetilde{\mathbf{K}}_{0}(\tilde{x}), & \widetilde{\mathbf{M}}(\tilde{x}, 0)=\widetilde{\mathbf{M}}_{0}(\tilde{x}),
\end{array}
$$

where $\widetilde{\mathbf{E}}_{0}(\tilde{x}), \widetilde{\mathbf{J}}_{0}(\tilde{x}), \widetilde{\mathbf{P}}_{0}(\tilde{x}), \widetilde{\mathbf{H}}_{0}(\tilde{x}), \widetilde{\mathbf{K}}_{0}(\tilde{x})$ and $\widetilde{\mathbf{M}}_{0}(\tilde{x})$ are some given functions.

To simplify the presentation, we first non-dimensionalize the Time-domain Lorentz model equations. Let us introduce the vacuum speed of light $C_{v}$, the vacuum impedance $Z_{0}$ :

$$
C_{v}=\frac{1}{\sqrt{\varepsilon_{0} \mu_{0}}} \approx 3 \times 10^{8} \mathrm{~m} / \mathrm{s}, \quad Z_{0}=\sqrt{\mu_{0} / \varepsilon_{0}} \approx 120 \pi \mathrm{ohms},
$$

and unit-free variables

$$
\begin{array}{lll}
t=\frac{C_{v} \tilde{t}}{L}, & x=\frac{\tilde{x}}{L}, & \Gamma_{e}=\frac{\tilde{\Gamma}_{e} L}{C_{v}}, \\
\Gamma_{m}=\frac{\tilde{\Gamma}_{m} L}{C_{v}}, & \omega_{p e}=\frac{\tilde{\omega_{p e}} L}{C_{v}}, & \omega_{p m}=\frac{\tilde{\omega}_{p m} L}{C_{v}}, \\
\omega_{e 0}=\frac{\tilde{\omega}_{e 0} L}{C_{v}}, & \omega_{m 0}=\frac{\tilde{\omega}_{m 0} L}{C_{v}}, & \mathbf{E}=\frac{\tilde{\mathbf{E}}}{Z_{0} H_{0}}, \\
\mathbf{H}=\frac{\tilde{\mathbf{H}}}{H_{0}}, & \mathbf{J}=\frac{L \tilde{\mathbf{J}}}{H_{0}}, & \mathbf{K}=\frac{L \mathbf{K}}{Z_{0} H_{0}}, \\
\mathbf{P}=\frac{C_{v} \tilde{\mathbf{P}}}{H_{0}}, & \mathbf{M}=\frac{C_{v} \tilde{\mathbf{M}}}{Z_{0} H_{0}}, &
\end{array}
$$

where $H_{0}$ is a unit magnetic field strength, and $L$ is a reference length (typically the wavelength of one interested object). 
It is not difficult to check that the equations can be written by

$$
\begin{aligned}
& \frac{\partial \mathbf{E}}{\partial t}+\mathbf{J}-\nabla \times \mathbf{H}=0 \\
& \frac{\partial \mathbf{H}}{\partial t}+\mathbf{K}+\nabla \times \mathbf{E}=0 \\
& \frac{\partial \mathbf{J}}{\partial t}+\Gamma_{e} \mathbf{J}+\omega_{e 0}^{2} \mathbf{P}-\omega_{p e}^{2} \mathbf{E}=0 \\
& \frac{\partial \mathbf{K}}{\partial t}+\Gamma_{m} \mathbf{K}+\omega_{m 0}^{2} \mathbf{M}-\omega_{p m}^{2} \mathbf{H}=0 \\
& \frac{\partial \mathbf{P}}{\partial t}=\mathbf{J} \\
& \frac{\partial \mathbf{M}}{\partial t}=\mathbf{K} .
\end{aligned}
$$

In the rest of this paper, our discussion is based on the non-dimensionalized form (2.2a)(2.2f).

\section{The semi-discrete DG method}

Normally, taking the Lagrange polynomials of order $N$ as trial functions, we approximate the solution at the $p$ nodal points within each element as

$$
\mathbf{A}^{k}(\mathbf{x}, t) \approx \mathbf{A}_{p}^{k}(\mathbf{x}, t)=\sum_{j=1}^{p} \mathbf{A}^{k}\left(\mathbf{x}_{j}, t\right) \mathbf{L}_{j}(\mathbf{x})=\sum_{j=1}^{p} \mathbf{A}_{j}^{k}(t) \mathbf{L}_{j}(\mathbf{x}),
$$

where $\mathbf{A}_{p}^{k}(\mathbf{x}, t)$ is the finite element approximation, and $\mathbf{A}^{k}\left(\mathbf{x}_{j}, t\right)$ represents the solution at nodal point $\mathbf{x}_{j}$. But with the increasing of the order of interpolation polynomials, serious oscillation phenomena will appear, which will increase the computational error and affect instabilities of computations. A natural solution to this problem is to seek an orthonormal basis a more suitable and computationally stable approach. In [22], The author choose the Legendre polynomials of order $N$ instead of the Lagrange polynomials and use the Legendre-Gauss-Lobatto (LGL) points as quadrature points. The distribution of these nodes in the standard triangle is shown in Fig. 1 for orders $N=2,3$.

In order to describe the system (2.2a)-(2.2f), we consider the uniform grid subdivision $T_{h}$ that partitions the domain $\Omega$ into $K_{T}$ disjoint triangular elements $T_{i}$ such that $\bar{\Omega}=$ $\bigcup_{i=1}^{K_{T}} T_{i}$. Furthermore, we denote $a_{i k}=T_{i} \cap T_{k}$ for an interior face between two adjacent elements $T_{i}$ and $T_{k}$, and $\mathbf{n}_{i k}$ for the unit normal vector pointed from $T_{i}$ to $T_{k}$. For any given element $T_{i}$, we denote $v_{i}$ for the set of all neighboring elements of $T_{i}$.

In DG methods, we consider the discretization space given by discontinuous piecewise polynomials of degree $N$ on each element, i.e.,

$$
\mathbf{V}_{h}=\left\{v_{h} \in\left(L^{2}(\Omega)\right)^{s}:\left.v_{h}\right|_{T_{i}} \in\left(\mathbf{P}_{N}\right)^{s}, \forall T_{i} \in T_{h}\right\}, \quad s=2,3 .
$$



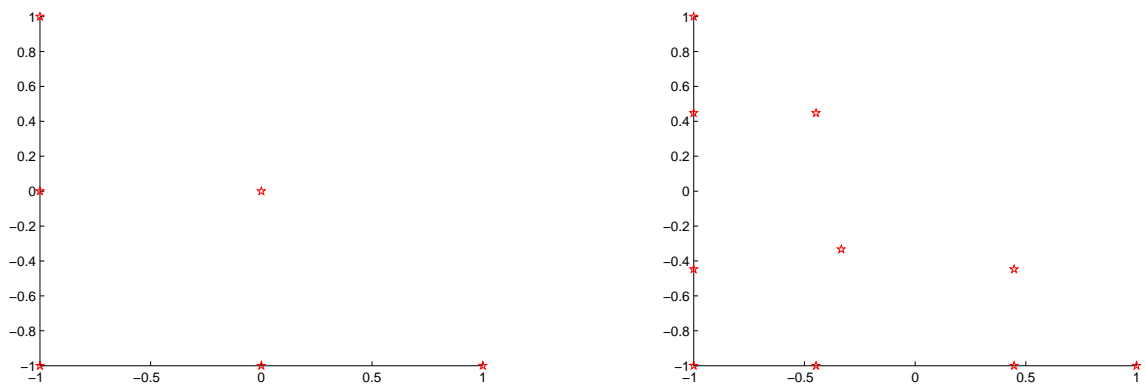

Figure 1: quadrature points for $N=2,3$.

Let us look at the semi-discrete solution $\mathbf{E}_{h}, \mathbf{H}_{h}, \mathbf{J}_{h}, \mathbf{K}_{h}, \mathbf{P}_{h}, \mathbf{M}_{h} \in \mathbf{C}\left(0, T ; \mathbf{V}_{h}\right)$ as a solution of the following weak formulation: For any $\mathbf{u}_{h}, \mathbf{v}_{h}, \varphi_{h}, \phi_{h}, \psi_{h}, \zeta_{h} \in \mathbf{V}_{h}$, and any element $T_{i} \in T_{h}$,

$$
\begin{aligned}
& \int_{T_{i}} \frac{\partial \mathbf{E}_{h}}{\partial t} \cdot \mathbf{u}_{h}-\int_{T_{i}} \mathbf{H}_{h} \cdot \nabla \times \mathbf{u}_{h}-\sum_{K \in v_{i}} \int_{a_{i k}} \mathbf{u}_{h} \cdot \mathbf{n}_{i k} \times\left\{\left\{\mathbf{H}_{h}\right\}\right\}_{i k}+\int_{T_{i}} \mathbf{J}_{h} \cdot \mathbf{u}_{h}=0, \\
& \int_{T_{i}} \frac{\partial \mathbf{H}_{h}}{\partial t} \cdot \mathbf{v}_{h}+\int_{T_{i}} \mathbf{E}_{h} \cdot \nabla \times \mathbf{v}_{h}+\sum_{K \in v_{i}} \int_{a_{i k}} \mathbf{v}_{h} \cdot \mathbf{n}_{i k} \times\left\{\left\{\mathbf{E}_{h}\right\}\right\}_{i k}+\int_{T_{i}} \mathbf{K}_{h} \cdot \mathbf{v}_{h}=0, \\
& \int_{T_{i}} \frac{\partial \mathbf{J}_{h}}{\partial t} \cdot \varphi_{h}+\Gamma_{e} \int_{T_{i}} \mathbf{J}_{h} \cdot \varphi_{h}+\omega_{e 0}^{2} \int_{T_{i}} \mathbf{P}_{h} \cdot \varphi_{h}-\omega_{p e}^{2} \int_{T_{i}} \mathbf{E}_{h} \cdot \varphi_{h}=0, \\
& \int_{T_{i}} \frac{\partial \mathbf{K}_{h}}{\partial t} \cdot \phi_{h}+\Gamma_{m} \int_{T_{i}} \mathbf{K}_{h} \cdot \phi_{h}+\omega_{m 0}^{2} \int_{T_{i}} \mathbf{M}_{h} \cdot \phi_{h}-\omega_{p m}^{2} \int_{T_{i}} \mathbf{H}_{h} \cdot \phi_{h}=0, \\
& \int_{T_{i}} \frac{\partial \mathbf{P}_{h}}{\partial t} \cdot \psi_{h}-\int_{T_{i}} \mathbf{J}_{h} \cdot \psi_{h}=0, \\
& \int_{T_{i}} \frac{\partial \mathbf{M}_{h}}{\partial t} \cdot \zeta_{h}-\int_{T_{i}} \mathbf{K}_{h} \cdot \zeta_{h}=0,
\end{aligned}
$$

hold true and are subject to the initial conditions:

$$
\begin{array}{lll}
\mathbf{E}_{h}(0)=\Pi_{2} \mathbf{E}_{0}, & \mathbf{J}_{h}(0)=\Pi_{2} \mathbf{J}_{0}, & \mathbf{P}_{h}(0)=\Pi_{2} \mathbf{P}_{0}, \\
\mathbf{H}_{h}(0)=\Pi_{2} \mathbf{H}_{0}, & \mathbf{K}_{h}(0)=\Pi_{2} \mathbf{K}_{0}, & \mathbf{M}_{h}(0)=\Pi_{2} \mathbf{M}_{0} .
\end{array}
$$

Recall that $\mathbf{E}_{0}, \mathbf{J}_{0}, \mathbf{M}_{0}, \mathbf{H}_{0}, \mathbf{K}_{0}$ and $\mathbf{M}_{0}$ are the given initial condition functions. For any function $\mathbf{v}_{h}$, we denote the average and jump through any internal face $a_{i k}$ as

$$
\left\{\mathbf{v}_{h}\right\}_{i k}=\frac{1}{2}\left(\mathbf{v}_{i}+\mathbf{v}_{k}\right), \quad\left[\left[\mathbf{v}_{h}\right]\right]_{i k}=\left(\mathbf{v}_{i}-\mathbf{v}_{k}\right),
$$

and the numerical flux ([18])

$$
\left\{\left\{\mathbf{H}_{h}\right\}\right\}_{i k}=\left\{\mathbf{H}_{h}\right\}_{i k}-\alpha \mathbf{n}_{i k} \times\left\{\mathbf{E}_{h}\right\}_{i k}, \quad\left\{\left\{\mathbf{E}_{h}\right\}\right\}_{i k}=\alpha \mathbf{n}_{i k} \times\left\{\mathbf{H}_{h}\right\}_{i k}+\left\{\mathbf{E}_{h}\right\}_{i k} .
$$


The parameter $\alpha$ in the numerical flux can be used to control dissipation; for example, taking $\alpha=0$ yields a nondissipative central flux and $\alpha=1$ results in the classic upwind flux. One is free, however, to take $\alpha$ to be any value in between.

Note that the perfectly conducting boundary condition is treated as $\left.\mathbf{E}_{k}\right|_{a_{i k}}=-\left.\mathbf{E}_{i}\right|_{a_{i k}}$ and $\left.\mathbf{H}_{k}\right|_{a_{i k}}=\left.\mathbf{H}_{i}\right|_{a_{i k}}$, which lead to

$$
\{\mathbf{E}\}_{i k}=0, \quad\{\mathbf{H}\}_{i k}=\mathbf{H}_{i} \mid a_{i k}, \quad \text { for any } a_{i k} \in \partial \Omega .
$$

Here and below we denote $\mathbf{E}_{i}=\left.\mathbf{E}_{h}\right|_{T_{i}}, \mathbf{H}_{i}=\left.\mathbf{H}_{h}\right|_{T_{i}}, \mathbf{J}_{i}=\left.\mathbf{J}_{h}\right|_{T_{i}}, \mathbf{K}_{i}=\left.\mathbf{K}_{h}\right|_{T_{i}}, \mathbf{P}_{i}=\left.\mathbf{P}_{h}\right|_{T_{i}}$ and $\mathbf{M}_{i}=\left.\mathbf{M}_{h}\right|_{T_{i}}$.

Denote the semi-discrete energy $\mathfrak{I}_{h}$ :

$$
\begin{aligned}
\mathfrak{S}_{h}(t)=\frac{1}{2}( & \left\|\mathbf{E}_{h}(t)\right\|_{0, \Omega}^{2}+\left\|\mathbf{H}_{h}(t)\right\|_{0, \Omega}^{2}+\frac{1}{\omega_{p e}^{2}}\left\|\mathbf{J}_{h}(t)\right\|_{0, \Omega}^{2} \\
& \left.\quad+\frac{1}{\omega_{p m}^{2}}\left\|\mathbf{K}_{h}(t)\right\|_{0, \Omega}^{2}+\frac{\omega_{e 0}^{2}}{\omega_{p e}^{2}}\left\|\mathbf{P}_{h}(t)\right\|_{0, \Omega}^{2}+\frac{\omega_{m 0}^{2}}{\omega_{p m}^{2}}\left\|\mathbf{M}_{h}(t)\right\|_{0, \Omega}^{2}\right),
\end{aligned}
$$

and a bilinear form $\aleph_{i}$ :

$$
\begin{aligned}
\aleph_{i}(\mathbf{E}, \mathbf{H})=- & \int_{T_{i}} \mathbf{H}_{i} \cdot \nabla \times \mathbf{i}_{h}-\sum_{K \in v_{i}} \int_{a_{i k}} \mathbf{E}_{h} \cdot \mathbf{n}_{i k} \times\left\{\left\{\mathbf{H}_{h}\right\}\right\}_{i k} \\
& +\int_{T_{i}} \mathbf{E}_{i} \cdot \nabla \times \mathbf{H}_{i}+\sum_{K \in v_{i}} \int_{a_{i k}} \mathbf{H}_{h} \cdot \mathbf{n}_{i k} \times\left\{\left\{\mathbf{E}_{h}\right\}\right\}_{i k} .
\end{aligned}
$$

Theorem 3.1. The energy $\mathfrak{I}_{h}$ is decreasing in time, i.e., $\mathfrak{I}_{h}(t) \leq \mathfrak{I}_{h}(0)$ for any $t \in[0, T]$.

Proof. Choosing $\mathbf{u}_{h}=\mathbf{E}_{h}, \mathbf{v}_{h}=\mathbf{H}_{h}, \varphi_{h}=\mathbf{J}_{h}, \phi_{h}=\mathbf{K}_{h}, \psi_{h}=\mathbf{P}_{h}, \zeta_{h}=\mathbf{M}_{h}$ in (3.1a)-(3.1f), and adding the results over all elements $T_{i} \in T_{h}$, we obtain

$$
\frac{d}{d t} \mathfrak{I}_{h}(t)+\frac{\Gamma_{e}}{\omega_{p e}^{2}}\left\|\mathbf{J}_{h}(t)\right\|_{0}^{2}+\frac{\Gamma_{m}}{\omega_{p m}^{2}}\left\|\mathbf{K}_{h}(t)\right\|_{0}^{2}+\sum_{i} \aleph_{i}(\mathbf{E}, \mathbf{H})=0
$$

Based on the definition of $\aleph_{i}$, integration by parts and the identity $(\mathbf{x} \times \mathbf{y}) \cdot \mathbf{z}=\mathbf{x} \cdot(\mathbf{y} \times \mathbf{z})$, we have

$$
\begin{aligned}
& \aleph_{i}(\mathbf{E}, \mathbf{H}) \\
= & \sum_{K \in v_{i}} \int_{a_{i k}} \mathbf{E}_{i} \cdot \mathbf{n}_{i k} \times \mathbf{H}_{i}+\sum_{K \in v_{i}} \int_{a_{i k}} \mathbf{E}_{i} \cdot\left\{\left\{\mathbf{H}_{i}\right\}\right\}_{i k} \times \mathbf{n}_{i k}-\sum_{K \in v_{i}} \int_{a_{i k}} \mathbf{H}_{i} \cdot\left\{\left\{\mathbf{E}_{i}\right\}\right\}_{i k} \times \mathbf{n}_{i k} \\
= & \sum_{K \in v_{i}} \int_{a_{i k}}\left[-\mathbf{E}_{i} \times \mathbf{H}_{i}+\mathbf{E}_{i} \times \frac{\mathbf{H}_{i}+\mathbf{H}_{k}}{2}-\mathbf{H}_{i} \times \frac{\mathbf{E}_{i}+\mathbf{E}_{k}}{2}\right] \cdot \mathbf{n}_{i k} \\
= & \frac{1}{2} \sum_{K \in v_{i}} \int_{a_{i k}}\left(\mathbf{E}_{i} \times \mathbf{H}_{k}+\mathbf{E}_{k} \times \mathbf{H}_{i}\right) \cdot \mathbf{n}_{i k} .
\end{aligned}
$$


Let (3.5) be summed up over all elements of $T_{h}$, we can obtain $\sum_{i} \aleph_{i}(\mathbf{E}, \mathbf{H})=0$. Combined with (3.4), we come to the conclusion $\frac{d}{d t} \mathfrak{s}_{h}(t) \leq 0$, which concludes the proof.

Lemma 3.1. ([22]) Assume that $u \in H^{p}(\Omega),(p>1 / 2)$, and that $u_{h}$ represents a piecewise polynomial interpolation of order $N$. Then

$$
\left\|u-u_{h}\right\|_{\Omega, q, h} \leq C \frac{h^{\sigma-q}}{N^{p-2 q-1 / 2}}|u|_{\Omega, \sigma, q}
$$

for $0 \leq q \leq \sigma$, and $\sigma=\min (N+1, p)$.

We have the following convergence result for the semi-discrete scheme (3.1a)-(3.1f).

Theorem 3.2. Assume that the domain $\Omega$ is parted uniformly, if $\mathbf{E}, \mathbf{H}, \mathbf{J}, \mathbf{K}, \mathbf{P}, \mathbf{M} \in \mathbf{C}^{0}([0, T]$; $\left.\left(\mathbf{H}^{p+1}(\Omega)\right)^{3}\right)$ for $p \geq 0$, then there exists a constant $C>0$ independent of $h$ such that

$$
\begin{gathered}
\max _{t \in[0, T]}\left(\left\|\mathbf{E}-\mathbf{E}_{\mathbf{h}}\right\|_{0, \Omega}+\left\|\mathbf{H}-\mathbf{H}_{\mathbf{h}}\right\|_{0, \Omega}+\left\|\mathbf{J}-\mathbf{J}_{\mathbf{h}}\right\|_{0, \Omega}+\left\|\mathbf{K}-\mathbf{K}_{\mathbf{h}}\right\|_{0, \Omega}+\left\|\mathbf{P}-\mathbf{P}_{\mathbf{h}}\right\|_{0, \Omega}\right. \\
\left.+\left\|\mathbf{M}-\mathbf{M}_{\mathbf{h}}\right\|_{0, \Omega}\right) \leq C h^{\sigma-1}\|(\mathbf{E}, \mathbf{H}, \mathbf{J}, \mathbf{K}, \mathbf{P}, \mathbf{M})\|_{\mathbf{C}^{0}\left([0, T] ;\left(\mathbf{H}^{p+1}(\Omega)\right)^{3}\right)},
\end{gathered}
$$

where $\sigma=\min (N+1, p)$.

Proof Let us introduce the notation $\overline{\mathbf{X}}_{h}=\Pi_{2} \mathbf{X}-\mathbf{X}$ and $\widetilde{\mathbf{X}}_{h}=\Pi_{2} \mathbf{X}-\mathbf{X}_{h}$ for $\mathbf{X}=$ E, H, J, K, P, M. Subtracting (3.1a)-(3.1f) , we have the error equations:

$$
\begin{aligned}
& \int_{T_{i}} \frac{\partial \widetilde{\mathbf{E}}_{h}}{\partial t} \cdot \mathbf{u}_{h}-\int_{T_{i}} \widetilde{\mathbf{H}}_{h} \cdot \nabla \times \mathbf{u}_{h}-\sum_{K \in v_{i}} \int_{a_{i k}} \mathbf{u}_{h} \cdot \mathbf{n}_{i k} \times\left\{\left\{\widetilde{\mathbf{H}}_{h}\right\}\right\}_{i k}+\int_{T_{i}} \widetilde{\mathbf{J}}_{h} \cdot \mathbf{u}_{h} \\
= & \int_{T_{i}} \frac{\partial \overline{\mathbf{E}}_{h}}{\partial t} \cdot \mathbf{u}_{h}-\int_{T_{i}} \overline{\mathbf{H}}_{h} \cdot \nabla \times \mathbf{u}_{h}-\sum_{K \in v_{i}} \int_{a_{i k}} \mathbf{u}_{h} \cdot \mathbf{n}_{i k} \times\left\{\left\{\overline{\mathbf{H}}_{h}\right\}\right\}_{i k}+\int_{T_{i}} \overline{\mathbf{J}}_{h} \cdot \mathbf{u}_{h}, \\
& \int_{T_{i}} \frac{\partial \widetilde{\mathbf{H}}_{h}}{\partial t} \cdot \mathbf{v}_{h}+\int_{T_{i}} \widetilde{\mathbf{E}}_{h} \cdot \nabla \times \mathbf{v}_{h}+\sum_{K \in v_{i}} \int_{a_{i k}} \mathbf{v}_{h} \cdot \mathbf{n}_{i k} \times\left\{\left\{\widetilde{\mathbf{E}}_{h}\right\}\right\}_{i k}+\int_{T_{i}} \widetilde{\mathbf{K}}_{h} \cdot \mathbf{v}_{h} \\
= & \int_{T_{i}} \frac{\partial \overline{\mathbf{H}}_{h}}{\partial t} \cdot \mathbf{v}_{h}+\int_{T_{i}} \overline{\mathbf{E}}_{h} \cdot \nabla \times \mathbf{v}_{h}+\sum_{K \in v_{i}} \int_{a_{i k}} \mathbf{v}_{h} \cdot \mathbf{n}_{i k} \times\left\{\left\{\overline{\mathbf{E}}_{h}\right\}\right\}_{i k}+\int_{T_{i}} \overline{\mathbf{K}}_{h} \cdot \mathbf{v}_{h}, \\
= & \int_{T_{i}} \frac{\partial \widetilde{\mathbf{J}}_{h}}{\partial t} \cdot \varphi_{h}+\Gamma_{e} \int_{T_{i}} \frac{\partial \overline{\mathbf{J}}_{h}}{\partial t} \cdot \varphi_{h}+\varphi_{e} \int_{T_{i}}^{2} \int_{T_{h}} \widetilde{\mathbf{P}}_{h} \cdot \varphi_{h}+\varphi_{e 0}^{2} \int_{T_{i}} \overline{\mathbf{P}}_{h} \cdot \varphi_{h}-\omega_{p e}^{2} \int_{T_{i}} \widetilde{\mathbf{E}}_{h} \cdot \varphi_{h} \cdot \varphi_{h}, \\
& \int_{T_{i}} \frac{\partial \widetilde{\mathbf{K}}_{h}}{\partial t} \cdot \phi_{h}+\Gamma_{m} \int_{T_{i}} \widetilde{\mathbf{K}}_{h} \cdot \phi_{h}+\omega_{m 0}^{2} \int_{T_{i}} \tilde{\mathbf{M}}_{h} \cdot \phi_{h}-\omega_{p m}^{2} \int_{T_{i}} \widetilde{\mathbf{H}}_{h} \cdot \phi_{h} \\
= & \int_{T_{i}} \frac{\partial \overline{\mathbf{K}}_{h}}{\partial t} \cdot \phi_{h}+\Gamma_{m} \int_{T_{i}} \overline{\mathbf{K}}_{h} \cdot \phi_{h}+\omega_{m 0}^{2} \int_{T_{i}} \overline{\mathbf{M}}_{h} \cdot \phi_{h}-\omega_{p m}^{2} \int_{T_{i}} \overline{\mathbf{H}}_{h} \cdot \phi_{h},
\end{aligned}
$$




$$
\begin{aligned}
& \int_{T_{i}} \frac{\partial \widetilde{\mathbf{P}}_{h}}{\partial t} \cdot \psi_{h}-\int_{T_{i}} \widetilde{\mathbf{J}}_{h} \cdot \psi_{h}=\int_{T_{i}} \frac{\partial \overline{\mathbf{P}}_{h}}{\partial t} \cdot \psi_{h}-\int_{T_{i}} \overline{\mathbf{J}}_{h} \cdot \psi_{h}, \\
& \int_{T_{i}} \frac{\partial \widetilde{\mathbf{M}}_{h}}{\partial t} \cdot \zeta_{h}-\int_{T_{i}} \widetilde{\mathbf{K}}_{h} \cdot \zeta_{h}=\int_{T_{i}} \frac{\partial \overline{\mathbf{M}}_{h}}{\partial t} \cdot \zeta_{h}-\int_{T_{i}} \overline{\mathbf{K}}_{h} \cdot \zeta_{h} .
\end{aligned}
$$

Choosing $\mathbf{u}_{h}=\widetilde{\mathbf{E}}_{h}, \mathbf{v}_{h}=\widetilde{\mathbf{H}}_{h}, \varphi_{h}=\widetilde{\mathbf{J}}_{h}, \phi_{h}=\widetilde{\mathbf{K}}_{h}, \psi_{h}=\widetilde{\mathbf{P}}_{h}, \zeta_{h}=\widetilde{\mathbf{M}}_{h}$ in (3.7a)-(3.7f), summing up the results over all elements $T_{i} \in T_{h}$, afterwards using the energy definition (3.2) and the projection property, we have

$$
\begin{aligned}
& \frac{d}{d t} \widetilde{\mathfrak{I}}_{h}(t)+\frac{\Gamma_{e}}{\omega_{p e}^{2}}\left\|\widetilde{\mathbf{J}}_{h}(t)\right\|_{0}^{2}+\frac{\Gamma_{m}}{\omega_{p m}^{2}}\left\|\widetilde{\mathbf{K}}_{h}(t)\right\|_{0}^{2}+\sum_{i} \aleph_{i}(\widetilde{\mathbf{E}}, \widetilde{\mathbf{H}}) \\
= & \sum_{i} \sum_{K \in v_{i}}\left[\int_{a_{i k}} \widetilde{\mathbf{E}}_{h} \cdot \mathbf{n}_{i k} \times\left\{\left\{\overline{\mathbf{H}}_{i}\right\}\right\}_{i k}+\int_{a_{i k}} \widetilde{\mathbf{H}}_{h} \cdot \mathbf{n}_{i k} \times\left\{\left\{\overline{\mathbf{E}}_{i}\right\}\right\}_{i k}\right] \\
\leq & \sum_{i}\left[\left\|\widetilde{\mathbf{E}}_{h}\right\|_{0, \partial T_{i}}\left\|\overline{\mathbf{H}}_{h}\right\|_{0, \partial T_{i}}+\left\|\widetilde{\mathbf{H}}_{h}\right\|_{0, \partial T_{i}}\left\|\overline{\mathbf{E}}_{h}\right\|_{0, \partial T_{i}}\right] \\
\leq & \sum_{i}\left[C h_{T_{i}}^{-\frac{1}{2}}\left\|\widetilde{\mathbf{E}}_{h}\right\|_{0, T_{i}} C h_{T_{i}}^{\sigma-\frac{1}{2}}\|\mathbf{H}\|_{p+1, T_{i}}+C h_{T_{i}}^{-\frac{1}{2}}\left\|\widetilde{\mathbf{H}}_{h}\right\|_{0, T_{i}} C h_{T_{i}}^{\sigma-\frac{1}{2}}\|\mathbf{E}\|_{p+1, T_{i}}\right] \\
\leq & \sum_{i}\left[C h_{T_{i}}^{\sigma-1}\left\|\widetilde{\mathbf{E}}_{h}\right\|_{0, T_{i}}\|\mathbf{H}\|_{p+1, T_{i}}+C h_{T_{i}}^{\sigma-1}\left\|\widetilde{\mathbf{H}}_{h}\right\|_{0, T_{i}}\|\mathbf{E}\|_{p+1, T_{i}}\right],
\end{aligned}
$$

where $\sigma$ is $\min (N+1, p)$, in the last step we used the trace theorem, the standard inverse inequality and interpolation error estimate.The proof is completed by using the Gronwall inequality and the fact $\sum_{i} \aleph_{i}(\mathbf{E}, \mathbf{H})=0$.

\section{Computational scheme}

\subsection{Spatial discretization}

We assume that the domain $\Omega$ is decomposed into tetrahedral (or triangular in 2D) elements $T_{k}$, and take electric field as an example, the numerical solution $\mathbf{E}_{k}$ is represented as

$$
\mathbf{E}_{k}(\mathbf{x}, t)=\sum_{j=1}^{\mathbf{N}_{n}} \mathbf{E}_{k}\left(\mathbf{x}_{j}, t\right) \mathbf{L}_{j}(\mathbf{x})=\sum_{j=1}^{\mathbf{N}_{n}} \mathbf{E}_{j}(t) \mathbf{L}_{j}(\mathbf{x}),
$$

where $\mathbf{L}_{j}(x)$ is the multivariate Lagrange interpolation polynomial of degree $\mathrm{N}$. Here we know $\mathbf{N}_{n}=\frac{1}{6}(N+1)(N+2)(N+3)$ in $3 \mathrm{D}$ and $\mathbf{N}_{n}=\frac{1}{2}(N+1)(N+2)$ in $2 \mathrm{D}$.

Superinducing the fixed source $\mathbf{f}$ in (2.2a), choosing $\mathbf{u}_{h}=\mathbf{L}_{i}(x)$ in semi-discrete solution (3.1a) and integrating over each element $T_{k}$, we obtain

$$
\begin{aligned}
& \int_{T_{k}}\left(\frac{\partial \mathbf{E}_{k}}{\partial t}+\mathbf{J}_{k}-\mathbf{f}_{k}\right) \mathbf{L}_{i}(x)-\mathbf{H}_{k} \cdot \nabla \times \mathbf{L}_{i}(x) d x \\
= & \int_{\partial T_{k}} \frac{1}{2} \hat{\mathbf{n}} \times\left(\left[\mathbf{H}_{k}\right]-\hat{\mathbf{n}} \times\left[\mathbf{E}_{k}\right]\right) \mathbf{L}_{i}(x) d x,
\end{aligned}
$$


where $\hat{\mathbf{n}}$ is an outward normal unit vector of $\partial \Omega_{k}$, $\left[\mathbf{E}_{k}\right]=\mathbf{E}_{k}^{+}+\mathbf{E}_{k}^{-}$, and $\left[\mathbf{H}_{k}\right]=\mathbf{H}_{k}^{+}+\mathbf{H}_{k}^{-}$. Here superscripts ' + ' and ' - ' refer to field values from the neighbor element and the local element itself, respectively. For this Maxwell's equations, we usually choose the upwind flux $(\alpha=1)$ [26].

Substituting (4.1) into (4.2), we obtain the elementwise equations for the electric field components

$$
\sum_{j=0}^{\mathbf{N}_{n}}\left(\mathbf{M}_{i j} \frac{d \mathbf{E}_{j}}{d t}-\mathbf{S}_{i j} \times \mathbf{H}_{j}-\mathbf{M}_{i j} \mathbf{S}_{E, j}\right)=\frac{1}{2} \sum_{l} \Phi_{i l} \cdot \hat{\mathbf{n}}_{l} \times\left(\left[\mathbf{H}_{l}\right]-\hat{\mathbf{n}}_{l} \times\left[\mathbf{E}_{l}\right]\right),
$$

where

$$
\mathbf{M}_{i j}=\left(\mathbf{L}_{i}(x), \mathbf{L}_{j}(x)\right)_{T_{k}}, \quad \mathbf{S}_{i j}=\left(\mathbf{L}_{i}(x), \nabla \mathbf{L}_{j}(x)\right)_{T_{k}}
$$

represent the local mass and stiffness matrices, respectively. Furthermore,

$$
\boldsymbol{\Phi}_{i l}=\left(\mathbf{L}_{i}(x), \mathbf{L}_{l}(x)\right)_{\partial T_{k}}
$$

represents the face-based mass matrix. Similarly, the elementwise equations for the magnetic field components

$$
\sum_{j=0}^{\mathbf{N}_{n}}\left(\mathbf{M}_{i j} \frac{d \mathbf{H}_{j}}{d t}+\mathbf{S}_{i j} \times \mathbf{E}_{j}+\mathbf{M}_{i j} \mathbf{S}_{H, j}\right)=\frac{1}{2} \sum_{l} \Phi_{i l} \cdot \hat{\mathbf{n}}_{l} \times\left(-\hat{\mathbf{n}}_{l} \times\left[\mathbf{H}_{l}\right]-\left[\mathbf{E}_{l}\right]\right) .
$$

We can rewrite (4.3) and (4.4) in a fully explicit form, while the constitutive equations (2.2c)-(2.2f) keep the same form. In summary, we have the following semi-discrete discontinuous Galerkin scheme:

$$
\begin{aligned}
\frac{d \mathbf{E}_{N}}{d t} & =\mathbf{M}^{-1} \mathbf{S} \times \mathbf{H}_{N}-\mathbf{J}_{N}+\mathbf{f}_{N}+\left.\frac{1}{2} \mathbf{M}^{-1} \mathbf{\Phi}\left(\hat{\mathbf{n}} \times\left(\left[\mathbf{H}_{N}\right]-\hat{\mathbf{n}} \times\left[\mathbf{E}_{N}\right]\right)\right)\right|_{\partial T_{k}}, \\
\frac{d \mathbf{H}_{N}}{d t} & =-\mathbf{M}^{-1} \mathbf{S} \times \mathbf{E}_{N}-\mathbf{K}_{N}+\mathbf{g}_{N}-\left.\frac{1}{2} \mathbf{M}^{-1} \mathbf{\Phi}\left(\hat{\mathbf{n}} \times\left(\hat{\mathbf{n}} \times\left[\mathbf{H}_{N}\right]+\left[\mathbf{E}_{N}\right]\right)\right)\right|_{\partial T_{k}}, \\
\frac{d \mathbf{J}_{N}}{d t} & =-\Gamma_{e} \mathbf{J}_{N}-\omega_{e 0}^{2} \mathbf{P}_{N}+\omega_{p e}^{2} \mathbf{E}_{N}, \\
\frac{d \mathbf{K}_{N}}{d t} & =-\Gamma_{m} \mathbf{K}_{N}-\omega_{m 0}^{2} \mathbf{M}_{N}+\omega_{p m}^{2} \mathbf{H}_{N}, \\
\frac{d \mathbf{P}_{N}}{d t} & =\mathbf{J}_{N}, \\
\frac{d \mathbf{M}_{N}}{d t} & =\mathbf{K}_{N} .
\end{aligned}
$$

\subsection{Time discretization}

The system (4.5a)-(4.5f) can be solved by various methods developed for a system of ordinary differential equations (ODE)

$$
\frac{d \mathbf{u}_{h}}{d t}=\Re\left(\mathbf{u}_{h}, t\right)
$$


where $\mathbf{u}_{h}$ is the vector of unknowns. In our realization, the classic low-storage five-stage fourth-order explicit Runge-Kutta method [12,22] presented in [19] was adopted:

$$
\begin{array}{ll}
\mathbf{p}^{(0)}=\mathbf{u}^{n}, & \mathbf{k}^{(0)}=0, \\
i \in[1, \ldots, 5]: & \left\{\begin{array}{l}
\mathbf{k}^{(i)}=a_{i} \mathbf{k}^{(i-1)}+\Delta t \Re_{h}\left(\mathbf{p}^{(i-1)}, t^{n}+c_{i} \Delta t\right), \\
\mathbf{p}^{(i)}=\mathbf{p}^{(i-1)}+b_{i} \mathbf{k}^{(i)},
\end{array}\right. \\
\mathbf{u}_{h}^{n+1}=\mathbf{p}^{(5)}, &
\end{array}
$$

where coefficients $a_{i}, b_{i}$ and $c_{i}$ are fixed constants given in Table 3.2 of [12]. Storage can be essential for low-storage schemes and large-scale computations need only two storage units per ODE variable by this method. The time-step restriction then can generally be taken as

$$
\Delta t \leq \Delta t_{\max }=C F L \frac{h_{k}}{c_{k}},
$$

where $h_{k}$ is the minimum edge length of all elements and $c_{k}$ is the maximum wave speed in the domain (see $[26,29]$ ). The scheme gives $(N+1)$ st-order convergence with the stability bound

$$
C F L=C \frac{1}{2 N+1}
$$

with $C=1$, as long as for a given spatial discretisation of polynomial order $\mathrm{N}$, the method has order $N+1$.

\section{Numerical results}

The implementation of our results is based on the information provided by [22]. For the sake of simplicity, here we only consider the implementation of 2D situation which is similar to the 3D situation. The following numerical experiment results were acquired using MATLAB 7.1 running on Lenovo Z485 laptop with $4 \mathrm{~GB}$ of RAM and $1.90 \mathrm{GHz}$ CPU for Time-domain Lorentz model equations in transverse electric (TE) format and transverse magnetic (TM) format.

\subsection{D TE case}

We define variables as $\mathbf{E}=\left(E_{x}, E_{y}, 0\right), \mathbf{H}=\left(0,0, H_{z}\right), \mathbf{J}=\left(J_{x}, J_{y}, 0\right), \mathbf{K}=\left(0,0, K_{z}\right), \mathbf{P}=$ $\left(P_{x}, P_{y}, 0\right), \mathbf{M}=\left(0,0, M_{z}\right)$ in $2 \mathrm{D}$ TE case where the subscripts ' $\mathrm{x}$ ', ' $\mathrm{y}$ ' and ' $\mathrm{z}$ ' denote the corresponding components.

To check the convergence rate obtained by our implementation, the following exact solutions are constructed with $\Gamma_{e}=\Gamma_{m}=2, \omega_{e 0}=\omega_{m 0}=\omega_{p e}=\omega_{p m}=1$ on domain $\Omega=(0,1)^{2}$. The exact solution to $(2.2 \mathrm{a})-(2.2 \mathrm{f})$ is 


$$
\begin{aligned}
& \mathbf{E}=\left(\begin{array}{c}
E_{x} \\
E_{y}
\end{array}\right)=e^{-t}\left(\begin{array}{c}
-\cos (\pi x) \sin (\pi y) \\
\sin (\pi x) \cos (\pi y)
\end{array}\right), \\
& H_{z}=e^{-t} \cos (\pi x) \cos (\pi y), \\
& \mathbf{J}=\left(\begin{array}{c}
J_{x} \\
J_{y}
\end{array}\right)=\left(-\frac{1}{2} t^{2}+t\right) e^{-t}\left(\begin{array}{c}
-\cos (\pi x) \sin (\pi y) \\
\sin (\pi x) \cos (\pi y)
\end{array}\right), \\
& K_{z}=\left(-\frac{1}{2} t^{2}+t\right) e^{-t} \cos (\pi x) \cos (\pi y), \\
& \mathbf{P}=\left(\begin{array}{c}
P_{x} \\
P_{y}
\end{array}\right)=\frac{1}{2} t^{2} e^{-t}\left(\begin{array}{c}
-\cos (\pi x) \sin (\pi y) \\
\sin (\pi x) \cos (\pi y)
\end{array}\right), \\
& M_{z}=\frac{1}{2} t^{2} e^{-t} \cos (\pi x) \cos (\pi y) .
\end{aligned}
$$

It is obvious that $\mathbf{E}$ satisfies the boundary condition $\hat{n} \times \mathbf{E}=0$ on $\partial \Omega$.

The corresponding source term $\mathbf{f}$ and $\mathbf{g}$ is given by:

$$
\begin{aligned}
& \mathbf{f}=\left(\begin{array}{l}
f_{x} \\
f_{y}
\end{array}\right)=\left(-\frac{1}{2} t^{2}+t-\pi-1\right)\left(\begin{array}{c}
-\cos (\pi x) \sin (\pi y) \\
\sin (\pi x) \cos (\pi y)
\end{array}\right), \\
& g_{z}=\left(-\frac{1}{2} t^{2}+t+2 \pi-1\right) \cos (\pi x) \cos (\pi y) .
\end{aligned}
$$

Table 1: $L^{2}$ and $L^{\infty}$ errors for electric field $E_{x}$ with linear basis function and $\tau=10^{-5}$ after 1 time step.

\begin{tabular}{||c|cccc||}
\hline Meshes & $L^{2}$ errors & order & $L^{\infty}$ errors & order \\
\hline 4 & $7.3057 \mathrm{e}-002$ & - & $2.2213 \mathrm{e}-005$ & - \\
8 & $1.8930 \mathrm{e}-002$ & 1.9484 & $1.2020 \mathrm{e}-005$ & 0.8860 \\
16 & $4.7749 \mathrm{e}-003$ & 1.9871 & $6.1259 \mathrm{e}-006$ & 0.9724 \\
32 & $1.1964 \mathrm{e}-003$ & 1.9968 & $3.0760 \mathrm{e}-006$ & 0.9939 \\
64 & $2.9927 \mathrm{e}-004$ & 1.9992 & $1.5380 \mathrm{e}-006$ & 0.9999 \\
\hline
\end{tabular}

Table 2: $L^{2}$ and $L^{\infty}$ errors for electric field $E_{x}$ with quadratic basis function and $\tau=10^{-5}$ after 1 time step.

\begin{tabular}{||c|cccc||}
\hline Meshes & $L^{2}$ errors & order & $L^{\infty}$ errors & order \\
\hline 4 & $1.2031 \mathrm{e}-003$ & - & $6.0683 \mathrm{e}-006$ & - \\
8 & $1.2484 \mathrm{e}-004$ & 3.2687 & $1.5893 \mathrm{e}-006$ & 1.9329 \\
16 & $1.4651 \mathrm{e}-005$ & 3.0910 & $4.0161 \mathrm{e}-007$ & 1.9845 \\
32 & $1.8000 \mathrm{e}-006$ & 3.0249 & $1.0043 \mathrm{e}-007$ & 1.9996 \\
64 & $2.2405 \mathrm{e}-007$ & 3.0061 & $2.4896 \mathrm{e}-008$ & 2.0122 \\
\hline
\end{tabular}

Results in Table 1-3 are obtained on the uniform triangular mesh with different basis functions after 1 time step with $\tau=10^{-5}$ for 2D-TE model. It demonstrates that the degree of error convergence is $N+1$ ( $N$ is the order of basis functions) for $L^{2}$ errors which coincide 
Table 3: $L^{2}$ and $L^{\infty}$ errors for electric field $E_{x}$ with cubic basis function and $\tau=10^{-5}$ after 1 time step.

\begin{tabular}{||ccccc||}
\hline Meshes & $L^{2}$ errors & order & $L^{\infty}$ errors & order \\
\hline 4 & $4.0258 \mathrm{e}-004$ & - & $9.6908 \mathrm{e}-007$ & - \\
8 & $2.5626 \mathrm{e}-005$ & 3.9736 & $1.2522 \mathrm{e}-007$ & 2.9521 \\
16 & $1.6090 \mathrm{e}-006$ & 3.9934 & $1.5755 \mathrm{e}-008$ & 2.9905 \\
32 & $1.0072 \mathrm{e}-007$ & 3.9978 & $2.0198 \mathrm{e}-009$ & 2.9636 \\
64 & $6.3365 \mathrm{e}-009$ & 3.9905 & $3.8863 \mathrm{e}-010$ & 2.3778 \\
\hline
\end{tabular}

Table 4: $L^{2}$ and $L^{\infty}$ errors for electric field $E_{x}$ with cubic basis function and $\tau=10^{-8}$ after 1000 time step.

\begin{tabular}{||ccccc||}
\hline Meshes & $L^{2}$ errors & order & $L^{\infty}$ errors & order \\
\hline 4 & $4.0258 \mathrm{e}-004$ & - & $9.6926 \mathrm{e}-007$ & - \\
8 & $2.5626 \mathrm{e}-005$ & 3.9736 & $1.2532 \mathrm{e}-007$ & 2.9513 \\
16 & $1.6090 \mathrm{e}-006$ & 3.9934 & $1.5786 \mathrm{e}-008$ & 2.9889 \\
32 & $1.0068 \mathrm{e}-007$ & 3.9983 & $1.9744 \mathrm{e}-009$ & 2.9992 \\
64 & $6.2942 \mathrm{e}-009$ & 3.9996 & $2.4616 \mathrm{e}-010$ & 3.0037 \\
\hline
\end{tabular}

with our theoretical analysis. The degradation of the order is produced in pace with the subdivision of the encryption in Table 3 . Table 4 comes from the experiment with smaller time step $\tau=10^{-8}$ for 1000 time steps so that we can compare the results acquired in Table 3 at the same ending time. Comparing Table 4 with Table 3, we see that the results obtained with the smaller time step $\tau=10^{-8}$ shows $\mathscr{O}\left(h^{4}\right)$ convergence rate for $L^{2}$ errors and $\mathscr{O}\left(h^{3}\right)$ convergence rate for $L^{\infty}$ errors very well, and the errors are more accurate than those obtained with $\tau=10^{-5}$. We consider that when $N=3, \tau=10^{-5}$ is not satisfied (4.6) with the decrease of $h$.

Now the following numerical examples are carried out in order to discuss the low and higher wave frequency in different $h$ and $N$. We suppose that $\Gamma_{e}=\Gamma_{m}=2 \omega \pi$, $\omega_{e 0}=\omega_{m 0}=\omega_{p e}=\omega_{p m}=\omega \pi$ on domain $\Omega=(0,1)^{2}$, the exact solution is:

$$
\begin{aligned}
& \mathbf{E}=\left(\begin{array}{c}
E_{x} \\
E_{y}
\end{array}\right)=e^{-\omega \pi t}\left(\begin{array}{c}
-\cos (\omega \pi x) \sin (\omega \pi y) \\
\sin (\omega \pi x) \cos (\omega \pi y)
\end{array}\right), \\
& H_{z}=e^{-\omega \pi t} \cos (\omega \pi x) \cos (\omega \pi y), \\
& \mathbf{J}=\left(\begin{array}{c}
J_{x} \\
J_{y}
\end{array}\right)=\left(-\frac{1}{2} \omega^{3} \pi^{3} t^{2}+\omega^{2} \pi^{2} t\right) e^{-\omega \pi t}\left(\begin{array}{c}
-\cos (\omega \pi x) \sin (\omega \pi y) \\
\sin (\omega \pi x) \cos (\omega \pi y)
\end{array}\right), \\
& K_{z}=\left(-\frac{1}{2} \omega^{3} \pi^{3} t^{2}+\omega^{2} \pi^{2} t\right) e^{-\omega \pi t} \cos (\omega \pi x) \cos (\omega \pi y), \\
& \mathbf{P}=\left(\begin{array}{c}
P_{x} \\
P_{y}
\end{array}\right)=\frac{1}{2} \omega^{2} \pi^{2} t^{2} e^{-\omega \pi t}\left(\begin{array}{c}
-\cos (\omega \pi x) \sin (\omega \pi y) \\
\sin (\omega \pi x) \cos (\omega \pi y)
\end{array}\right), \\
& M_{z}=\frac{1}{2} \omega^{2} \pi^{2} t^{2} e^{-\omega \pi t} \cos (\omega \pi x) \cos (\omega \pi y) .
\end{aligned}
$$



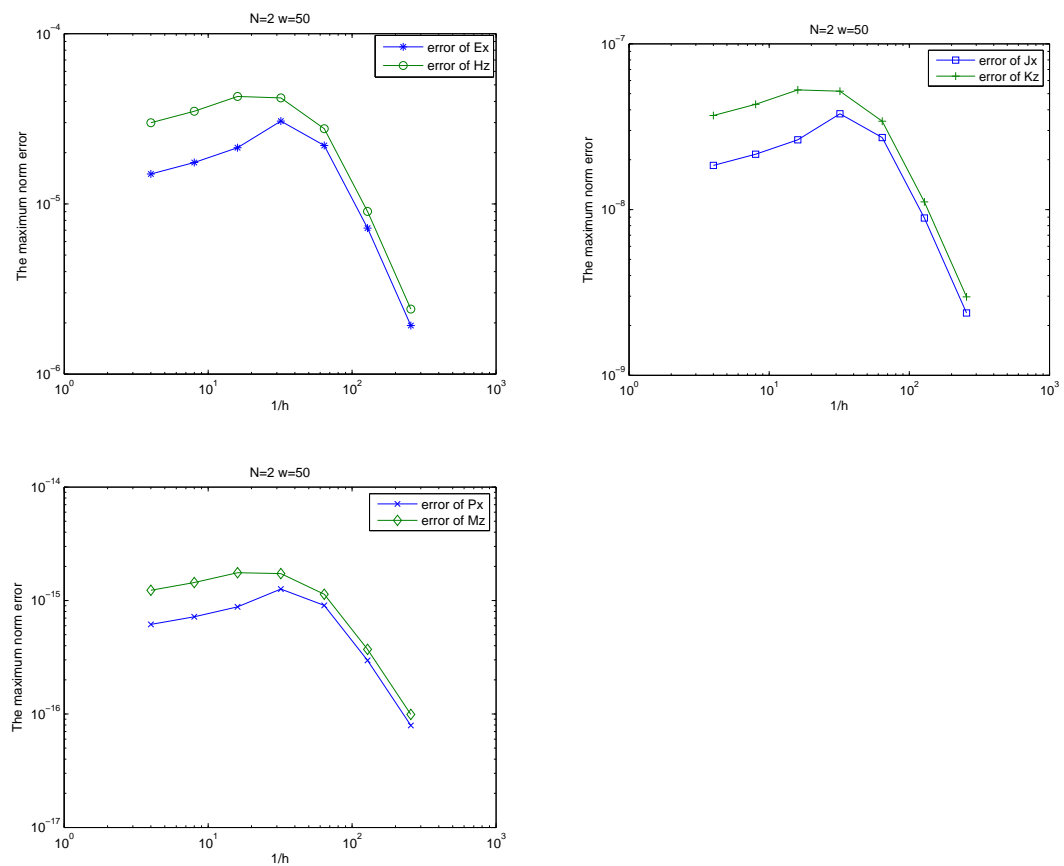

Figure 2: Errors of $\left\|E_{x}-E_{x, h}\right\|_{\infty, \Omega}$ and $\left\|H_{z}-H_{z, h}\right\|_{\infty, \Omega},\left\|J_{x}-J_{x, h}\right\|_{\infty, \Omega}$ and $\left\|K_{z}-K_{z, h}\right\|_{\infty, \Omega},\left\|P_{x}-P_{x, h}\right\|_{\infty, \Omega}$ and $\left\|M_{z}-M_{z, h}\right\|_{\infty, \Omega}$ for $N=2, w=50, \tau=10^{-8}$ at 10 time steps.

It is obvious that $\mathbf{E}$ satisfies the boundary condition $\hat{n} \times \mathbf{E}=0$ on $\partial \Omega$.

The corresponding source term $\mathbf{f}$ and $\mathbf{g}$ is given by:

$$
\begin{aligned}
& \mathbf{f}=\left(\begin{array}{l}
f_{x} \\
f_{y}
\end{array}\right)=\left(-\frac{1}{2} \omega^{3} \pi^{3} t^{2}+\omega^{2} \pi^{2} t-2 \omega \pi\right) e^{-\omega \pi t}\left(\begin{array}{c}
-\cos (\omega \pi x) \sin (\omega \pi y) \\
\sin (\omega \pi x) \cos (\omega \pi y)
\end{array}\right), \\
& g_{z}=\left(-\frac{1}{2} \omega^{3} \pi^{3} t^{2}+\omega^{2} \pi^{2} t+\omega \pi\right) e^{-\omega \pi t} \cos (\omega \pi x) \cos (\omega \pi y) .
\end{aligned}
$$

In the numerical results, we first show the dependence of the convergence of $\| E_{x}-$ $E_{x, h} \|_{\infty, \Omega}$ and $\left\|H_{z}-H_{z, h}\right\|_{\infty, \Omega}\left\|J_{x}-J_{x, h}\right\|_{\infty, \Omega},\left\|K_{z}-K_{z, h}\right\|_{\infty, \Omega},\left\|P_{x}-P_{x, h}\right\|_{\infty, \Omega}$ and $\left\|M_{z}-M_{z, h}\right\|_{\infty, \Omega}$ on polynomial order $\mathrm{N}$ and mesh size h. On one hand, Fig. 1 display the above six of these errors for $w=50, N=2 \tau=10^{-8}$ after 10 time steps. We find that the errors and mesh size $\mathrm{h}$ have a linear relationship as the mesh of encryption. Fig. 3 shows the error comparison by different polynomial approximations $(N=1,2,3)$. Fig. 4 displays the $L^{2}$ errors and $L^{\infty}$ errors for $E_{x}$ by fixed polynomial approximations for different wave frequency $w=10,20,30,40,50$. Now the total Dofs can be 1310720 for solving $\mathbf{E}_{h}$ when $N=3, w=50, K_{T}=131072$ in Fig 3. The results support the theoretical analysis.

\subsection{D TM case}

The following exact solutions are constructed for the 2D TM case which means $\mathbf{E}=$ $\left(0,0, E_{z}\right), \mathbf{H}=\left(H_{x}, H_{y}, 0\right), \mathbf{J}=\left(0,0, J_{z}\right), \mathbf{K}=\left(K_{x}, K_{y}, 0\right), \mathbf{P}=\left(0,0, P_{z}\right), \mathbf{M}=\left(M_{x}, M_{y}, 0\right)$. 

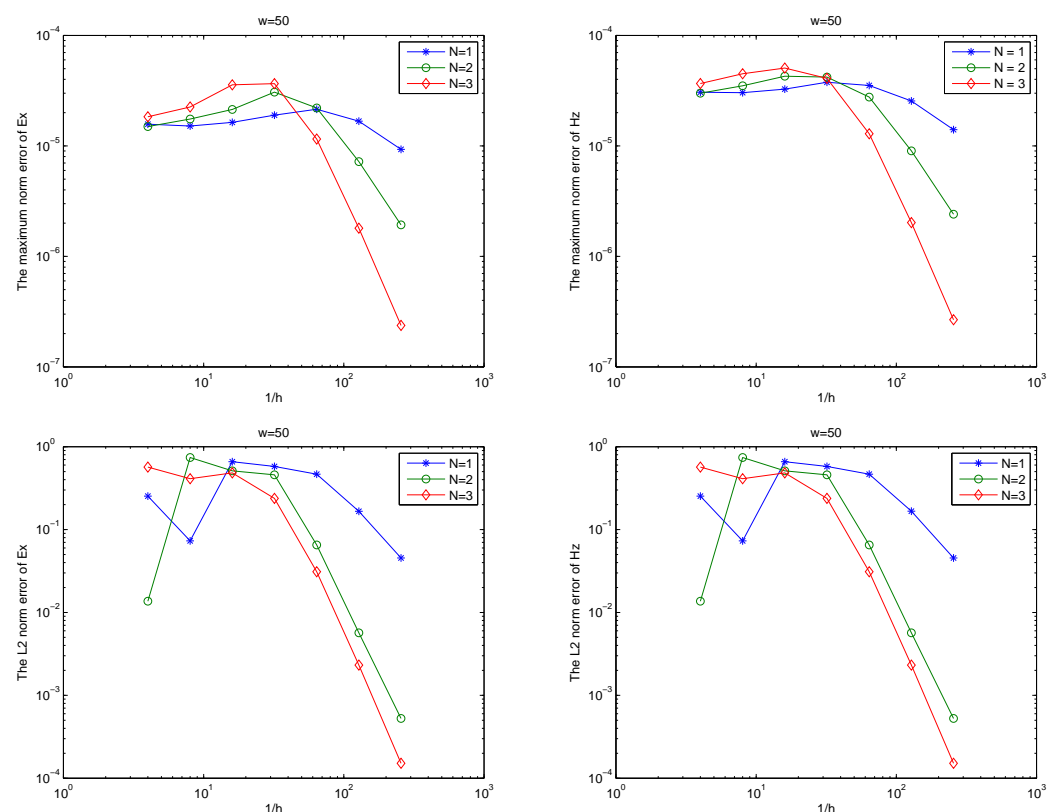

Figure 3: $L^{\infty}$ errors of $E_{x}$ and $H_{z}$ for $w=50$ by different polynomial approximations $(N=1,2,3)$ (top two); $L^{2}$ errors of $E_{x}$ and $H_{z}$ for $w=50$ by different polynomial approximations $(N=1,2,3)$ (bottom two).
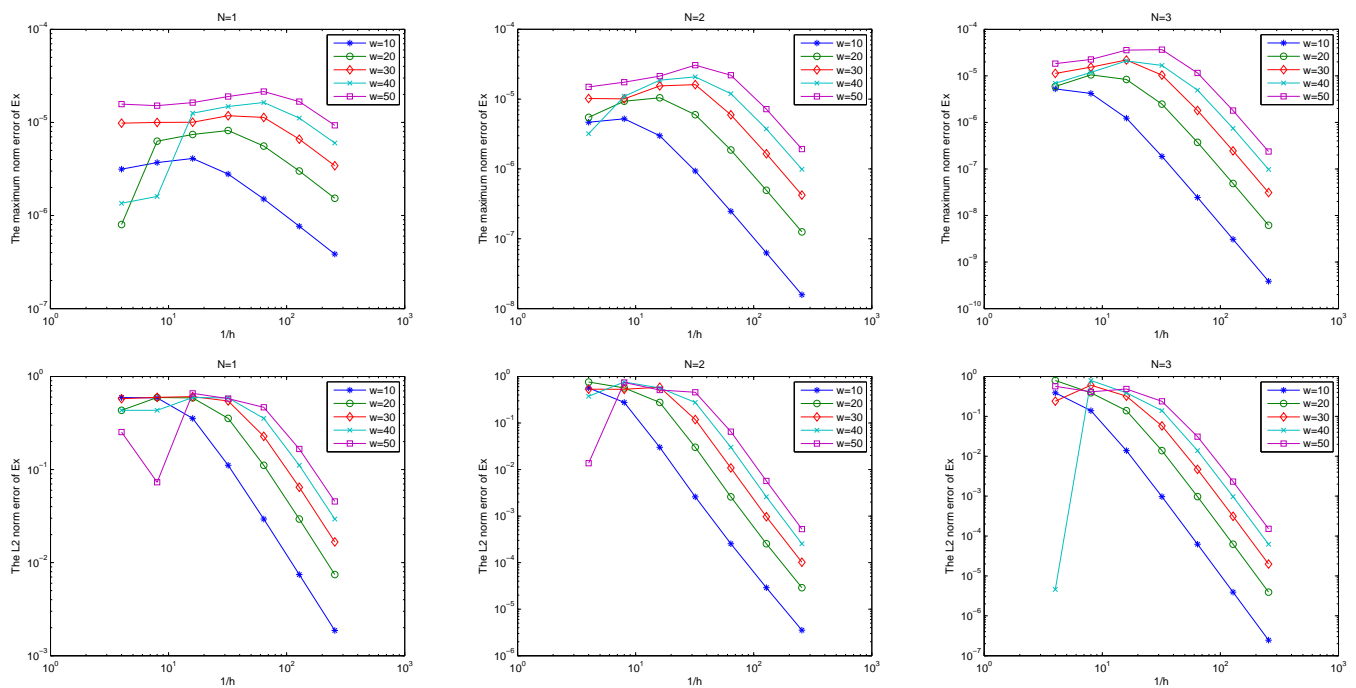

Figure 4: $L^{\infty}$ errors for $E_{x}$ by fixed polynomial approximations for $w=10,20,30,40,50$ (top); $L^{2}$ errors for $E_{x}$ by fixed polynomial approximations for $w=10,20,30,40,50$ (bottom).

More specifically, we assume that

$$
\Gamma_{e}=\Gamma_{m}=2, \quad \omega_{e 0}=\omega_{m 0}=\omega_{p e}=\omega_{p m}=1 .
$$


Table 5: $L^{2}$ errors for electric field $E_{z}$ and magnetic field $H_{x}$ with linear basis function and $h=2500 \tau^{2}$.

\begin{tabular}{||c|ccccc||}
\hline$\tau$ & $\mathrm{h}$ & errors for Ez & order & errors for Hx & order \\
\hline 0.01 & 0.25 & $7.0183 \mathrm{e}-002$ & - & $7.3487 \mathrm{e}-002$ & - \\
0.005 & 0.0625 & $4.6981 \mathrm{e}-003$ & 3.9010 & $4.8168 \mathrm{e}-003$ & 3.9313 \\
\hline
\end{tabular}

Table 6: $L^{2}$ errors for electric field $E_{z}$ and magnetic field $H_{x}$ with cubic basis function and $h=2500 \tau$.

\begin{tabular}{||c|ccccc||}
\hline$\tau$ & $\mathrm{h}$ & errors for Ez & order & errors for Hx & order \\
\hline 0.0001 & 0.25 & $4.0232 \mathrm{e}-004$ & - & $4.0268 \mathrm{e}-004$ & - \\
0.00005 & 0.0625 & $2.5613 \mathrm{e}-005$ & 3.9734 & $2.5633 \mathrm{e}-005$ & 3.9736 \\
\hline
\end{tabular}

on domain $\Omega=(0,1)^{2}$. The exact solution to (2.2a)-(2.2f) is

$$
\begin{aligned}
& \mathbf{H}=\left(\begin{array}{l}
H_{x} \\
H_{y}
\end{array}\right)=e^{-t}\left(\begin{array}{c}
\sin (\pi x) \cos (\pi y) \\
-\cos (\pi x) \sin (\pi y)
\end{array}\right), \\
& E_{z}=e^{-t} \sin (\pi x) \sin (\pi y), \\
& \mathbf{K}=\left(\begin{array}{l}
K_{x} \\
K_{y}
\end{array}\right)=\left(-\frac{1}{2} t^{2}+t\right) e^{-t}\left(\begin{array}{c}
\sin (\pi x) \cos (\pi y) \\
-\cos (\pi x) \sin (\pi y)
\end{array}\right), \\
& J_{z}=\left(-\frac{1}{2} t^{2}+t\right) e^{-t} \sin (\pi x) \sin (\pi y), \\
& \mathbf{M}=\left(\begin{array}{c}
M_{x} \\
M_{y}
\end{array}\right)=\frac{1}{2} t^{2} e^{-t}\left(\begin{array}{c}
\sin (\pi x) \cos (\pi y) \\
-\cos (\pi x) \sin (\pi y)
\end{array}\right), \\
& P_{z}=\frac{1}{2} t^{2} e^{-t} \sin (\pi x) \sin (\pi y) .
\end{aligned}
$$

It is obvious that $\mathbf{E}$ satisfies the boundary condition $\hat{n} \times \mathbf{E}=0$ on $\partial \Omega$.

The corresponding source term $\mathbf{f}$ and $\mathbf{g}$ is given by:

$$
\begin{aligned}
& \mathbf{g}=\left(\begin{array}{l}
g_{x} \\
g_{y}
\end{array}\right)=\left(-\frac{1}{2} t^{2}+t+\pi-1\right)\left(\begin{array}{c}
\sin (\pi x) \cos (\pi y) \\
-\cos (\pi x) \sin (\pi y)
\end{array}\right), \\
& f_{z}=\left(-\frac{1}{2} t^{2}+t-2 \pi-1\right) \sin (\pi x) \sin (\pi y) .
\end{aligned}
$$

Numerical solutions for $E_{z}\left(H_{x}\right)$ and the corresponding pointwise errors obtained with cubic basis function and $\tau=10^{-8}\left(\tau=10^{-10}\right)$ at the end of 10,000 time steps are presented in Fig. 5 (Fig. 6). Table 5-6 shows the results about the time step convergence. It validates the convergence $\mathscr{O}\left(\tau^{4}\right)$ which coincides with our theoretical analysis. Table 7 lists the results with cubic basis function and $\tau=10^{-10}$ after 10000 steps. It shows that the algorithm is quite efficient by considering that the CPU time. The long time stability and the rapidity of NDG operation is validated. 
Table 7: $L^{\infty}$ errors with cubic basis function and $\tau=10^{-10}$ after 10000 steps.

\begin{tabular}{||c|ccccc||}
\hline meshes & 4 & 8 & 16 & 32 & 64 \\
\hline$H_{x}$ & $9.6954 \mathrm{e}-008$ & $1.2540 \mathrm{e}-008$ & $1.5811 \mathrm{e}-009$ & $1.9795 \mathrm{e}-010$ & $2.5067 \mathrm{e}-011$ \\
$E_{z}$ & $1.0816 \mathrm{e}-007$ & $1.4078 \mathrm{e}-008$ & $1.7773 \mathrm{e}-009$ & $2.2268 \mathrm{e}-010$ & $2.8056 \mathrm{e}-011$ \\
$K_{x}$ & $4.8477 \mathrm{e}-014$ & $6.2701 \mathrm{e}-015$ & $7.9056 \mathrm{e}-016$ & $9.8976 \mathrm{e}-017$ & $1.2649 \mathrm{e}-017$ \\
$J_{z}$ & $5.4081 \mathrm{e}-014$ & $7.0393 \mathrm{e}-015$ & $8.8869 \mathrm{e}-016$ & $1.1134 \mathrm{e}-016$ & $1.4029 \mathrm{e}-017$ \\
$M_{x}$ & $1.6159 \mathrm{e}-020$ & $2.0901 \mathrm{e}-021$ & $2.6353 \mathrm{e}-022$ & $3.2994 \mathrm{e}-023$ & $4.2580 \mathrm{e}-024$ \\
$P_{z}$ & $1.8027 \mathrm{e}-020$ & $2.3465 \mathrm{e}-021$ & $2.9624 \mathrm{e}-022$ & $3.7111 \mathrm{e}-023$ & $4.6727 \mathrm{e}-024$ \\
CPU(s) & 115.7140 & 299.0448 & 354.5498 & 1033.0154 & 4521.4125 \\
\hline
\end{tabular}
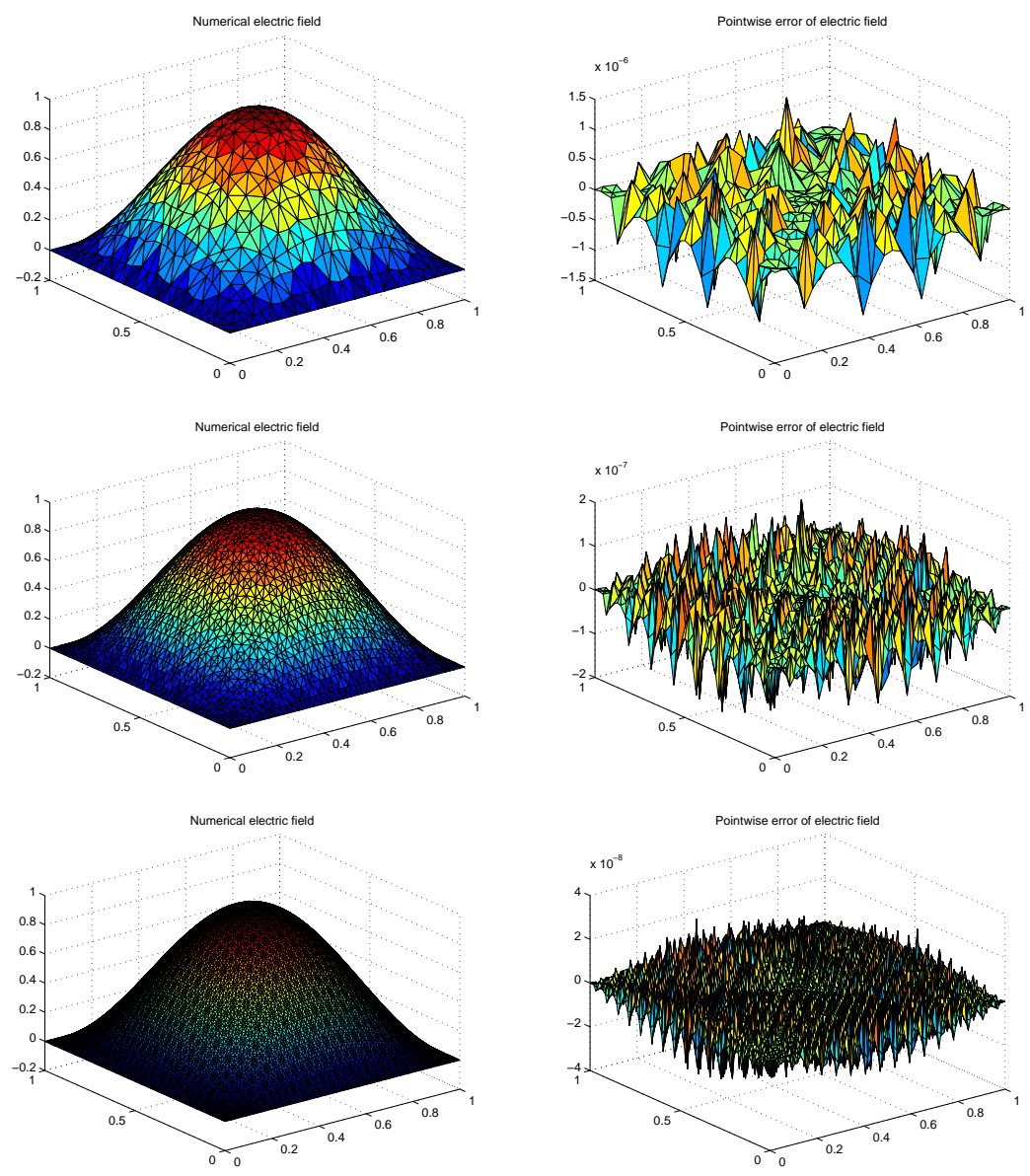

Figure 5: Results obtained with cubic basis function and $\tau=10^{-8}$ after 10000 time steps. Top row (with $h=\frac{1}{8}$ ): contour plot of $E_{z}$ (left) and its pointwise error (right); middle row (with $h=\frac{1}{16}$ ): contour plot of $E_{z}$ (left) and its pointwise error (right); bottom row (with $h=\frac{1}{32}$ ): contour plot of $E_{z}$ (left) and its pointwise error (right). 

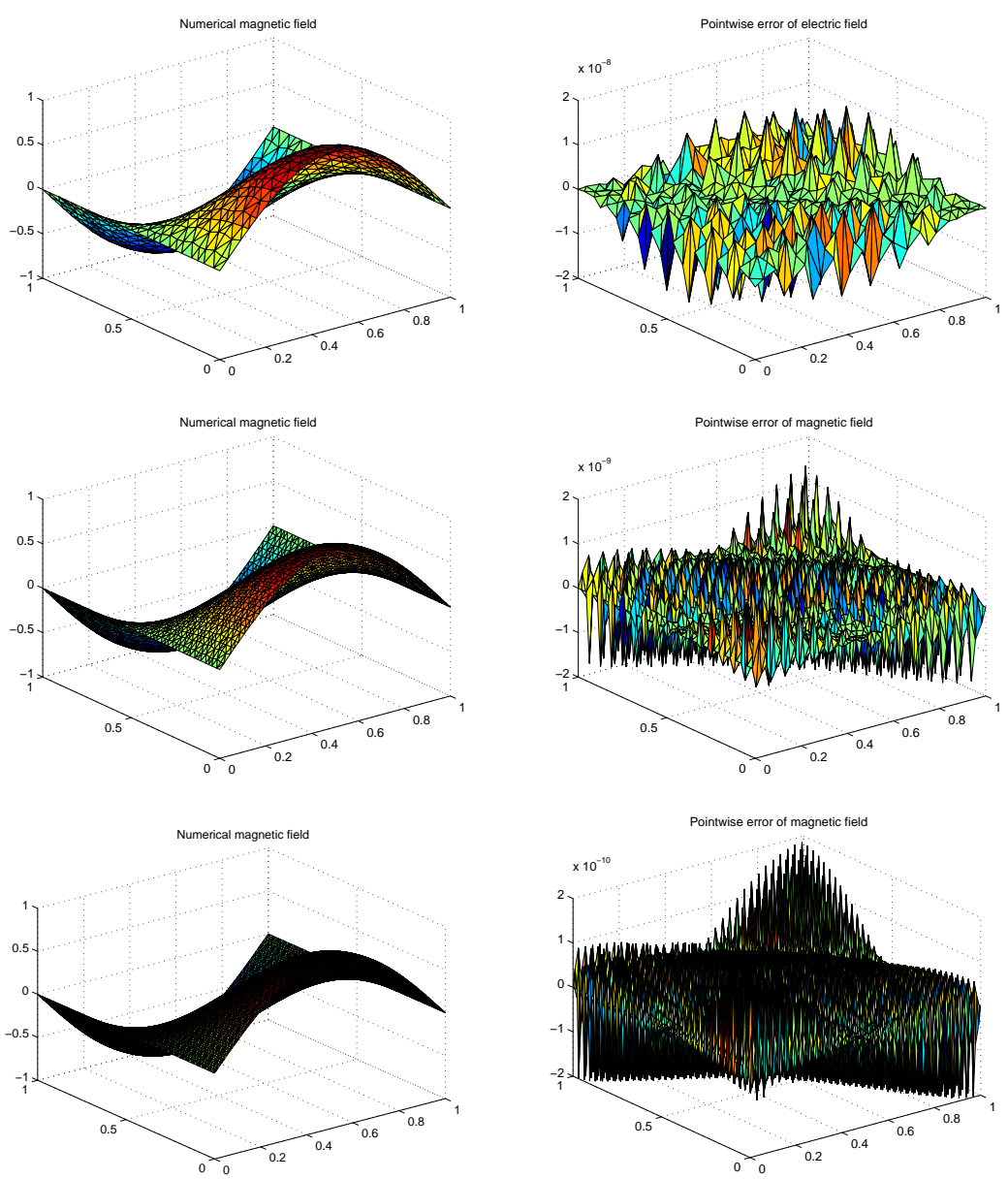

Figure 6: Results obtained with cubic basis function and $\tau=10^{-10}$ after 10000 time steps. Top row (with $\mathrm{h}=\frac{1}{8}$ ): contour plot of $H_{x}$ (left) and its pointwise error (right); middle row (with $\mathrm{h}=\frac{1}{16}$ ): contour plot of $H_{x}$ (left) and its pointwise error (right); bottom row (with $\mathrm{h}=\frac{1}{32}$ ): contour plot of $H_{x}$ (left) and its pointwise error (right).

\section{Conclusions}

In this paper, we provide NDG method to approximate Time-domain Lorentz model equations in meta-materials, where the upwind flux and Low-storage five-stage fourth-order explicit Runge-Kutta method are employed in spatial and time discrete scheme, respectively. We give the numerical stability and error estimate. Numerical experiments for TE case and TM case in low and higher wave frequency support the theoretical analysis.

Acknowledgments The first author is supported by NSFC. China (NOs. 11201501, 1157 1389) and the Program for Innovation Research in Central University of Finance and Economics. The second author is Supported by NSFC. China (Grant Nos. 11471296, 
11101384), and the third author is supported in part by Defense Industrial Technology Development Program (B1520133015).

\section{References}

[1] B. Cockburn, G. E. Karniadakis and C. W. Shu, Discontinuous Galerkin Methods: Theory, Computation and Applications, Springer, Berlin, 2000.

[2] D. N. ARnold, F. Brezzi, B. Cockburn and L. D. Marini, Unified analysis of discontinuous Galerkin methods for elliptic problems, SIAM J. Numer. Anal., 39 (2002), pp. 1749-1779.

[3] F. X. Giraldo, J. S. Hesthaven and T. Warburton, Nodal high-order discontinuous Galerkin methods for the spherical shallow water equations, J. Comput. Phys., 181 (2002), pp. 499-525.

[4] H. LiU AND J. YAN, The direct discontinuous Galerkin (DDG) methods for diffusion problems, SIAM J. Numer. Anal., 47 (2009), pp. 675-698.

[5] J. QIU, B. C. KHOO AND C. W. SHu, A numerical study for the performance of the Runge-Kutta discontinuous Galerkin method based on different numerical fluxes, J. Comput. Phys., 212 (2006), pp. 540-565.

[6] S. Sun, B. RiviéRe AND M. F. Wheeler, A combined mixed finite element and discontinuous Galerkin method for miscible displacement problem in porous media, Recent Progress in Computational and Applied PDES, Springer, 2003, pp. 323-352.

[7] B. CockBURn, F. LI, C. W. SHU, Locally divergence-free discontinuous Galerkin methods for the Maxwell equations, J. Comput. Phys., 194 (2004), pp. 588-610.

[8] E. T. Chung, P. Ciarlet JR. and T. F. Yu, Convergence and super convergence of staggered discontinuous Galerkin methods for the three-dimensional Maxwell's equations on Cartesian grids, J. Comput. Phys., 235 (2013), pp. 14-31.

[9] V. Dolean, H. FAHS, L. Fezoui AND S. LANTERI, Locally implicit discontinuous Galerkin method for time domain electromagnetics, J. Comput. Phys., 229 (2010), pp. 512-526.

[10] L. Fezoui, S. Lanteri, S. Lohrengel and S. Piperno. Convergence and stability of a discontinuous Galerkin time-domain methods for the 3D heterogeneous Maxwell equations on unstructured meshes, Modél. Math. Anal. Numér., 39(6) (2005), pp. 1149-1176.

[11] M. J. Grote And A. Schneebeli, D. SchötzAu, Interior penalty discontinuous Galerkin method for Maxwell's equations: energy norm error estimates, J. Comput. Appl. Math., 204 (2007), pp. 375-386.

[12] J. S. Hesthaven and T. Warburton, Nodal high-order methods on unstructured grids. I. Timedomain solution of Maxwell's equations, J. Comput. Phys, 181 (2002), pp. 186-221

[13] P. Houston, I. Perugia, A. Schneebeli And D. Schötzau, Interior penalty method for the indefinite time-harmonic Maxwell equations, Numer. Math., 100 (2005), pp. 485-518.

[14] E. Montseny, S. Pernet, X. Ferriéres And G. Cohen, Dissipative terms and local time-stepping improvements in a spatial high order discontinuous Galerkin scheme for the time-domain Maxwell's equations, J. Comput. Phys., 227 (2008), pp. 6795-6820.

[15] T. Lu, P. Zhang and W. CaI, Discontinuous Galerkin methods for dispersive and lossy Maxwell's equations and PML boundary conditions, J. Comput. Phys., 200 (2004), pp. 549-580.

[16] S. LANTERI AND C. SCHEID, Convergence of a discontinuous Galerkin scheme for the mixed time domain Maxwell's equations in dispersive media, IMAJ. Numer. Anal., 33 (2013), pp. 432-459.

[17] B. WANG, Z. XIE AND Z. ZhANG, Error analysis of a discontinuous Galerkin method for Maxwell equations in dispersive media, J. Comput. Phys., 229 (2010), pp. 552-8563.

[18] J. LI, J. W. WATERS AND E. A. MACHORRo, An implicit leap-frog discontinuous Galerkin method for the time-domain Maxwell's equations in metamaterials, Comput. Methods Appl. Mech. Eng., 223-224 (2012), pp. 43-54. 
[19] J. LI, Development of discontinuous Galerkin methods for Maxwell's equations in metamaterials and perfectly matched layers, J. Comput. Appl. Math., 236 (5)(2011), pp. 950-961.

[20] J. Li, Numerical convergence and physical fidelity analysis for Maxwell's equations in metamaterials, Comput. Methods Appl. Mech. Eng., 198 (2009), pp. 3161-3172.

[21] J. Li And Y. Huang, Time-Domain Finite Element Methods for Maxwell's Equations in Metamaterials, Springer Ser. Comput. Math., Springer, 43 (2013).

[22] J. S. Hesthaven and T. Warburton, Nodal Discontinuous Galerkin Methods: Algorithms, Analysis, and Applications, Springer, New York, 2008.

[23] Y. HuANG, J. LI AND W. YANG, Modeling Backward wave propagation in metamaterials by the finite element time domain method, SIAM J. Sci. Comput., 35(1) (2013), pp. B248-B274.

[24] W. YANG, Y. HuANG AND J. LI, Developing a time-domain finite element method for the Lorentz metamaterial model and applications, J. Sci. Comput., 68(2) (2016), pp. 438-463.

[25] H. Y. DuAn, F. JiA, P. Lin AND C. E. Roger TAN, The local L2 projected C0 finite element method for Maxwell problem, SIAM J. Numer. Anal., 47(2) (2009), pp. 1274-1303.

[26] D. Sármany, M. A. Botchev and J. J. W. van DER VegT, Dispersion and dissipation eerror in high-order Runge-Kutta discontinuous Galerkin discretisations of the Maxwell equations, J. Sci. Comput., 33 (2007), pp. 47-74.

[27] M. H. Carpenter and C. A. Kennedy, Fourth Order 2N-Storage Runge-Kutta Scheme, NASATM-109112, NASA Langley Research Center, 1994.

[28] J. H. Williamson, Low-storage Runge-Kutta schemes, J. Comput. Phys., 35(1) (1980), pp. 4856.

[29] M. H. Chen, B. CockBuRn And F. ReITich, High-order RKDG methods for computational electromagnetics, J. Sci. Comput., 22-23(1-3) (2005), pp. 205-226.

[30] J. Li And J. S. Hesthaven, Analysis and application of the nodal discontinuous Galerkin method for wave propagation in metamaterials, J. Comput. Phys., 258 (2014), pp. 915-930.

[31] Y. HuAng, J. LI AND W. YANG, Solving metamaterial Maxwell's equations via a vector wave integro-differential equation, Comput. Math. Appl., 63 (2012), pp. 1597-1606.

[32] J. Li AND Z. ZHANG, Unified analysis of time domain mixed finite element methods rof maxwell's equations in dispersive media, J. Comput. Math., 28(5) (2010), pp. 693-710. 